Ann. Biol. anim. Bioch. Biophys., I962, 2 (I), 47-50.

\title{
RÉSERVES SPERMATIQUES ÉPIDIDYMAIRES CHEZ LE VERRAT
}

\author{
G. SINGH (1) \\ Station de Recherches de Physiologie animale, \\ Centre national de Recherches zootechniques, Jouy-en-Josas (Seine-et-Oise).
}

SOMMAIRE

I.e nombre total de spermatozoïdes dans l'épididyme a été déterminé pour 8 Verrats de race Large White âgés de $\mathrm{I}-2$ ans après homogénéisation pendant $\mathrm{I}$ minute et macération à $+5^{\circ} \mathrm{C}$ pendant 96 heures. Ce nombre qui cst en moyenne de $95,4 \cdot 10^{\circ}$ spermatozoïdes par épididyme varie de 68,0 à $143,9 \cdot 10^{9} ; 54,4 \mathrm{p} .100$ des spermatozoïdes sont situés dans la queve de l'épididyme. La production quotidienne moyenne est de 13,6, I $_{4}, 6 \cdot 10^{9}$ spermatozoïdes.

\section{INTRODUC'TION}

L'estimation des réserves spermatiques épididymaires présente une importance particulière chez le Verrat. Le nombre moyen de spermatozoïdes par éjaculat est en effet extrêmement élevé chez cet animal puisqu'il atteint parfois Ioo milliards.

Chez le Bélier et le Taureau, pour un éjaculat contenant en moyenne 3 à 6 milliards de spermatozoïdes, les réserves spermatiques épididymaires sont respectivement de I30 à r6o milliards (Polovefva, I938; Chang, I945; Ortavant, I952) et de 50 à 60 milliards de spermatozoïdes (AlmoUist et HALE, I956 ; OrTAVAN'T, I959). Or chez le Verrat, les deux seuls travaux portant sur cette question indiquent que ces réserves sont constituées par I75 à 200 milliards de spermatozoïdes (NovOcELJEV, I95I; KENNELLY, I960). Devant une telle disproportion, par rapport aux autres animaux domestiques, entre la totalité des réserves spermatiques et la fraction de celles-ci mobilisée par un éjaculat, il nous a semblé important de reprendre l'étude de ce problème puisqu'il conditionne la fréquence des collectes.

(1) Adresse actuelle : Division of animal Genetics, Indian veterinary Research Institute, Izatnagar, U. P. India. 


\section{MATÉRIEL E'T MÉTHODES}

L'appareil génital de 8 Verrats de race Large White âgés de I-2 ans ont été obtenus dans un abattoir local pour cette étude. Chaque épididyme est séparé en trois fractions : la tête, le corps et la queue, après avoir été débarrassé de l'albuginée et de la plus grande partie de tissu conjonctif séparant les différents lobes. Chaque partie est alors découpée en petits fragments de 1 à $2 \mathrm{~cm}$ et dilué dans du sérum physiologique dans la proportion d'environ I : 4 .

Chaque partie d'un épididyme, alternativement le droit et le gauche est homogénéisée deux fois, pendant 30 secondes chaque fois, dans un Turmix. Le volume final de l'homogénat est amené à environ r litre. Afin d'étudier l'influence de la technique d'homogénéisation sur le nombre de spermatozoïdes comptés, l'autre épididyme fut traité différemment. A cet effet, chaque partie est homogénéisée de la même façon que précédemment pendant i minute au total puis la plus grande partie du surnageant est prélevée. Après addition d'une nouvelle quantité de sérum physiologique, le sédiment est soumis à une nouvelle homogénéisation identique à la précédente et le surnageant à nouveau prélevé. Cette opération est répétée une nouvelle fois puis tous les surnageants et le sédiment sont rassemblés. Le temps total d'homogénéisation pour ces épididymes est donc de 3 minutes.

Tous les homogénats sont gardés dans une chambre froide à $+5^{\circ} \mathrm{C}$. Des numérations de spermatozoïdes à l'hématimètre sont effectuées tous les jours sur deux parties aliquotes de chaque homogénat au cours de la macération. La connaissance du volume total de l’homogénat permet de détrerminer le nombre total de spermatozoïdes présent dans l'homogénat.

\section{RÉSULTATS}

Les résultats (tableau I et 2 ) permettent de se rendre compte que la technique de préparation des homogénats a une grande importance sur le nombre total de spermatozoïdes comptés. Dans tous les cas la meilleure durée de macération a été de 96 heures, puis de $7^{2}$ heures. Par ailleurs, lorsque la durée d'homogénéisation n'est que de $\mathrm{I}$ minute, le nombre de spermatozoïdes comptés par gramme de tissu épididymaire (I,2I4 $\cdot \mathrm{IO}^{9}$ ) est significativement plus élevé que lorsque la durée d'homogénéisation est de 3 minutes $\left(I, 006 \cdot I O^{9}\right)$, même si on soustrait le surnageant de 1'homogénéiseur après chaque minute d'homogénéisation.

\section{TABLEAU I}

Nombre moyen $\left(\mathrm{I}^{9}\right)$ de spermatozö̈des dans un épididyme de Verrat en fonction de différents facteurs techniques

\begin{tabular}{|c|c|c|c|}
\hline \multirow{2}{*}{$\begin{array}{c}\text { Durée } \\
\text { de macération }\end{array}$} & \multicolumn{2}{|c|}{ Durée d'homogénéisation } & \multirow{2}{*}{$\begin{array}{l}\text { Nombre } \\
\text { de données }\end{array}$} \\
\hline & 1 minute & 3 minutes & \\
\hline $2 / 4 h$ & $71,0 \div 7,1$ & $65,7+6,9$ & 8 \\
\hline $48 \mathrm{~h}$ & $75,2+6,3$ & $62,5 \pm 7,6$ & 8 \\
\hline $72 \mathrm{~h}$ & $81,6 \pm 6,0$ & $68,9 \pm 4,6$ & 8 \\
\hline $96 \mathrm{~h}$ & $95,4=8,3$ & $78,2 \pm 9,1$ & 8 \\
\hline
\end{tabular}

Le nombre de spermatozoïdes présents dans l'épididyme sera donc celui fourni par la meilleure technique : 96 heures de macération après une homogénéisation de I minute. 
TABLEAU 2

Analyse de variance du nombre de spermatozoïdes dans l'épididyme.

\begin{tabular}{|c|c|c|c|c|}
\hline Source de variation & Degré de liberté & Somme des carrés & Carré moyen & $\mathrm{F}$ \\
\hline Totale .............. & 63 & 29188 & & \\
\hline Temps d'homogénéisation. & 1 & 2201 & 2201 & Significatif \\
\hline Temps de macération .... & 3 & 3417 & 1139 & Significatif \\
\hline Côté de l'épididyme ..... & 1 & 39 & 39 & \\
\hline Animaux..$\ldots \ldots \ldots \ldots$ & 7 & $20 \quad 032$ & 2862 & Significatif \\
\hline Erreur ............... & 51 & 3496 & 68 & \\
\hline
\end{tabular}

Dans ces conditions le nombre moyen de spermatozoïdes par épididyme est de $95,4 \cdot 10^{9}$ mais il peut varier très largement puisque nous avons trouvé qu'il pouvait être aussi faible que $68,0 \cdot \mathrm{IO}^{9}$ et aussi élevé que $\mathrm{I} 43,3 \cdot \mathrm{IO}^{9}$. Ces spermatozoïdes se trouvent en grande partie dans la queue de l'épididyme $(54,4$ p. Ioo) alors que la tête et le corps ne renferment respectivement que I8,7 et 26,9 p. Ioo (tableau 3).

\section{TABLEAU 3}

Nombre moyen de spermatozoïdes dans les diffórentes parties de l'épididyme de Verrat

\begin{tabular}{|c|c|c|c|}
\hline Partie de l'épididyme & Nombre de $\operatorname{spz}\left(10^{9}\right)$ & $\%$ du total des spz & Nombre de donnćes \\
\hline Tête................. & $17,8 \pm 2,4$ & 18,7 & 8 \\
\hline 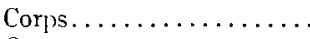 & $25,7 \pm 3,9$ & 26,9 & 8 \\
\hline Queue ............... & $51,9 \pm 5,8$ & $54,{ }^{\prime}$ & 8 \\
\hline 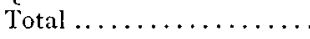 & $95,4 \pm 8,3$ & 100 & 8 \\
\hline
\end{tabular}

\section{DISCUSSION}

Cette étude préliminaire nous montre que chez le Verrat comme chez le Bélier, la durée d'homogénéisation ne doit pas dépasser $\mathrm{I}$ minute (ORTAvanT, I958). Dans ces conditions nos résultats coïncident assez bien avec ceux obtenus par NovOSELJCEV (I95I) et par KENNELLY (I960). Ces auteurs ont trouvé respectivement que les réserves spermatiques épididymaires totales étaient constituées par $\mathrm{I}_{7} 5^{-200} \cdot \mathrm{IO}^{9}$ et $\mathrm{I} 93 \cdot \mathrm{IO}^{9}$ spermatozoïdes alors que nous trouvons I9o,8. IO $^{9}$ chez nos animaux.

Nous avons trouvé auparavant que la durée de passage des spermatozoïdes dans l'épididyme est de I4 jours (SingH, I962). On peut donc estimer que, chez le Verrat, la production quotidienne de spermatozoïdes est en moyenne de $190,8.10^{9} / 14$, soit $\mathrm{I} 3,6 \cdot \mathrm{IO}^{9}$ spermatozoïdes (de 9,7 à $20,4 \cdot \mathrm{IO}^{9}$ spermatozoïdes). Ainsi un éjaculat est constitué par la production spermatique de 3 à 4 jours. On peut donc provisoirement conclure qu'un espacement de 3-4 jours entre deux collectes est nécessaire. Ceci coïncide assez bien avec les résultats obtenus dans la pratique des centres d'insémination artificielle porcine et par divers chercheurs (MC KEnziE, MrLier et Bauguess, r938 ; TURKheimer et al., I957). 


\section{REMERCIEMENTS}

L'auteur remercie R. ORTATANT, Directeur de Recherches à l'Institut national de la Recherche agronomique pour lui avoir suggéré ce travail et l'avoir guidé tout au long de sa réalisation.

Ce travail a pu être effectué grâce à deux bourses du Gouvernement français.

Reçu en janvier 1962.

\section{SUMMARY}

\section{SPERM RESERVES IN THE EPIDIDYMIS OF TILE BOAR}

$I^{0}$ The sperm reserves in the epididymis were determined in 8 pairs of the epididymis of young Boars aged $\mathrm{I}-2$ years.

$2^{6}$ Eight epididymis (4 right epididymis and 4 left epididymis) were homogenised for twice in one minute ( 30 seconds +30 seconds) in a Turmix blender. The three parts, head, body and the tail were homogenised separately.

$3^{0}$ The remaining 8 epididymis (4 right epididynis +4 left epididymis) were homogenised in the same way but for 3 minutes. After one minute homogenisation ( 30 seconds +30 seconds) the fluid was removed and the tissue was taken for homogenisation in physiological saline. This procedure was repeated for the third homogenisation.

$4^{\mathrm{e}}$ The macerated tissues were stored for 96 hours at $5^{\circ} \mathrm{C}$ in a cooling chamber.

$5^{\circ}$ The estimations of sperm concentration of each part of the epididymis for all macerated tissues were made at $24,48,72$ and 96 hours of storage. From these figures and the total volume of the macerated tissue of each part, the total sperm reserves in that portion was determined.

$6^{\circ}$ The best results were obtained with one minute of homogenisation and at 96 hours storage.

$7^{\circ}$ Results of the estimates revealed that epididymal sperm reserves ranged from 68.0 to $143.9 \cdot 10^{9}$ with a mean of $95.4 \cdot 10^{9}$ sperm per epididymis or $198.8 \cdot 10^{9}$ sperm per pair of epididymis on one minute maceration and 96 hours storage.

8. On a basis of percentage of the total sperm reserves in the epididynis, the caput, the corpus and the cauda contained 18.7 p. I0o, 26.9 p. 100 and 54.4 p. 100 respectively.

$9^{0}$ The estimated sperm production per day in Boar was 13.6 billions.

\section{RÉFÉRENCES BIBLIOGRAPHIQUES}

Almouist J. O., Hale E. B., 1956. An approach to the measurement of sexual behaviour and semen production of dairy Bulls. 3rd Int. Congr. Reprod., (('ambridge), 50-59.

Chang: N. C., I945. The sperm production of adult Rams in relation to frequency of semen collection. J. Agr. Sci., 35, 243-246.

KENXEI.LY J. J., I060. Spermatogenesis in Boar. I. -- The testicular and epididymal spermatozoan reserves. II. - Estimated potential daily production of spermatozoa. Dissert. M. Sc. Cornell Univ.

MIC Kenzie fi. F., Mirler J. C., Barguess L. C., 1938. The reproductive organs and semen of Boar. Res. Bull. Uni. Miss., 279, 55-6o.

NovoselJCEV D. V., I95I. The quantity of spermatozoa in the Boars epididymis and principles governing it. Sovetik. Zootech., 6, 76-83, in Anim. Breed. Abstr., 20, $5^{8}$.

ORtavant R., I952. Recherches quantitatives sur la spermatogenèse et les réserves spermatiques du Bélier. 2nd Int. Congr. Physiol. Patho. Anim. Reprod. Art. Ins. (Copenhagen), 63-69.

Ortavant R., I959. Déroulement et durée du cycle spermatogénétique chez le Bélier (première partie). Ann. Zootech., 8, 183-249.

Poloveleva V. V., 1938. Vitesse de transit et durée de maturation des spermatozoïdes dans l'épididyne du Bélier (en russe). Dok. Akad. Selj. Skokhoz. Nauk, 15-16, 43-52, in Anim. Breed. Abstr., 7, 227.

Singh $C_{i}$, ig62 Durée de passage dans l'épididyme des spematozoïdes de Verrat marqués au ${ }^{32} \mathrm{P}$. Ann. Biol. anim. Bioch. Biophys., 2, 43-46.

Tirkheiver A. R., Youvg D. C., Foote R. H., 1957. Semen production in young Boars collected once a day versus three days. $J$. Anim. Sci, 16, in 1o, (Abstr.). 